\title{
3D Revolute Joint with Clearance in Multibody Systems
}

\author{
Narendra Akhadkar ${ }^{1(\varpi)}$, Vincent Acary ${ }^{2}$, and Bernard Brogliato ${ }^{2}$ \\ 1 Schneider Electric, 37 Quai Paul-Louis Merlin, 38000 Grenoble, France \\ narendra.akhadkar@schneider-electric.com \\ 2 INRIA Grenoble Rhône-Alpes and Laboratoire Jean Kuntzman, \\ University Grenoble-Alpes, 655 avenue de l'Europe, 38334 Saint-Ismier, France \\ \{vincent. acary, bernard.brogliato\}@inria.fr
}

\begin{abstract}
The aim of this paper is to understand the influence of radial and axial clearance in the revolute joints on the overall performance of a circuit breaker. A circuit breaker mechanism is made of seven links, seven revolute joints with clearance in both radial and axial direction, four unilateral contacts with friction, and it has forty-two degrees of freedom. The Moreau-Jean nonsmooth contact dynamics (NSCD) numerical method is used to perform the simulations. The numerical results are validated by careful comparisons with experimental data.
\end{abstract}

Keywords: Joint clearance · Unilateral constraints · Coulomb's friction $\cdot$ Impacts $\cdot$ Circuit breaker

\section{Introduction}

A miniature circuit breaker is a device that switches and/or protects the lowest common distributed voltage in an electrical system. It is designed to protect conductors and insulation from damage due to overload and short circuit. Usually, the performance of these mechanisms is not as desired, due to the manufacturing tolerances on links, clearances in the joints and the assembly tolerances. The spatial revolute joint with clearance in both axial and radial direction adds five extra degrees of freedom into the system. Compared to planar mechanisms, spatial mechanisms can generate more complicated functions with the same number of links. Most of the previous work is focused on the radial clearance in the planar and spatial revolute joints $[8,10,11]$. However more recently the influence of the axial clearance in the revolute joint has been studied in $[15,17]$.

Most of the mechanisms in the Schneider Electric company use frictional contacts and the compliant models cannot correctly model the sticking condition. In the nonsmooth contact dynamic (NSCD) approach, the interaction of the colliding bodies is modeled with multiple frictional unilateral constraints $[12,13]$. The unilateral constraints are described by set-valued force laws in normal and tangential directions. The normal contact law is based on Signorini's condition while the tangential contact law is based on Coulomb's friction law. Careful 
comparisons between numerical results obtained with the NSCD approach, and experimental data are reported in $[14,16]$, while the use of the NSCD approach for systems with clearances is also advocated in [5,9]. They demonstrate that the numerical schemes and the model used in this article, though they can be improved, possess very good forecast capabilities.

Our objective is to study the influence of initial conditions and the out-ofplane motion, i.e. the polarization effect ${ }^{1}$ in the three dimensional case. Another objective is to develop a time efficient virtual test bench using the INRIA opensource simulation software SICONOS ${ }^{2}$. Our aim is to understand the influence of clearance in the revolute joints on the overall behavior of the C-60 miniature circuit breaker where human safety must be guaranteed. To validate the simulation model, experiments are carried-out on the prototype samples and the results are compared with the simulations.

\section{Formulation of the Nonsmooth Dynamical Systems}

\subsection{Normal and Tangential Contact Laws}

Let us consider two non overlapping bodies (see Fig. 1), a potential contact point between two bodies is given by the closest points $C_{A}$ and $C_{B}$. A local frame is defined at the potential contact point by $\left(\mathrm{N}, \mathrm{T}_{1}, \mathrm{~T}_{2}\right)$. The gap $g_{\mathrm{N}}$ is defined a the signed distance between the two potential contacting points $C_{A}$ and $C_{B}$. The contact force, denoted by $r=\left(r_{\mathrm{N}}, r_{\mathrm{T}}\right)^{\top} \in \mathbb{R}^{3}$. Due to the impenetrability assumption one has $g_{\mathrm{N}} \geqslant 0$. We also neglect adhesive effects so that $r_{\mathrm{N}} \geqslant 0$. If $r_{\mathrm{N}}>0$ then we impose $g_{\mathrm{N}}=0$, and when $g_{\mathrm{N}}>0$, the normal contact force must

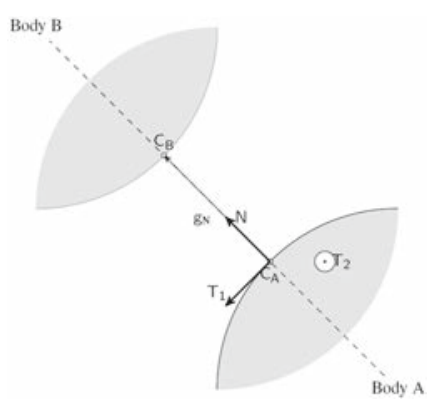

Fig. 1. Contact local frame.

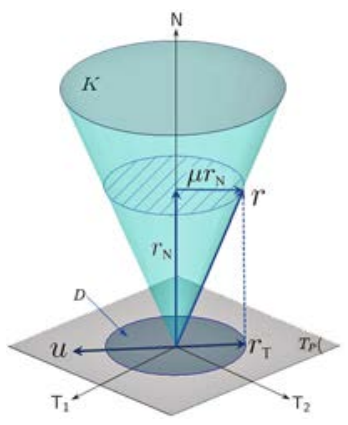

Fig. 2. 3D Coulomb's friction cone, sliding case.

\footnotetext{
${ }_{1}^{1}$ The polarization effect is created by two aspects: the presence of radial clearance in the revolute joint and the forces acting on the parts.

Definition 1. Polarization is the contact position between the two parts under the influence of an external force in an equilibrium stage.

2 http://siconos.gforge.inria.fr/.
} 
vanish, i.e. $r_{\mathrm{N}}=0$ (no magnetic or distance forces) $[1,3,6]$. These conditions yield a complementarity condition denoted compactly as:

$$
0 \leqslant g_{\mathrm{N}} \perp r_{\mathrm{N}} \geqslant 0 .
$$

The normal contact law at the velocity level is expressed as:

$$
0 \leqslant u_{\mathrm{N}} \perp r_{\mathrm{N}} \geqslant 0, \quad \text { if } g_{\mathrm{N}}=0 .
$$

The tangential contact law is the Coulomb friction that constrain the contact force $r$ in the the friction cone (see Fig. 2)

$$
r \in K=\left\{r \in \mathbb{R}^{3},\left\|r_{\mathrm{T}}\right\| \leqslant \mu r_{\mathrm{N}}\right\} .
$$

The scalar $\mu \geqslant 0$ is the coefficient of friction. In case of sliding the tangential force $r_{\mathrm{T}}$ acts in direction opposite to the relative tangential velocity $u_{\mathrm{T}}$. If the relative tangential velocity $u_{\mathrm{T}}$ is zero then the bodies stick to each other (rolling without slipping). We introduce the modified relative velocity $\widehat{u}:=u+\mu\left\|u_{\mathrm{T}}\right\| \mathrm{N}$, then the Coulomb friction can be equivalently expressed as a second-order cone complementarity condition [4,7] if $g_{\mathrm{N}}=0$ :

$$
K^{*} \ni \widehat{u} \perp r \in K .
$$

The cone $K^{*}=\left\{v \in \mathbb{R}^{3} \mid r^{T} v \geqslant 0, \forall r \in K\right\}$ is the dual cone of $K$.

\subsection{Newton-Euler Formulation of the Equation of Motion}

Let us consider a mechanical system subjected to $m$ constraints, with $m_{e}$ holonomic bilateral constraints $h^{\alpha}(q)=0, \alpha \in \mathscr{E} \subset \mathbb{N}, m_{i}$ unilateral constraints $g_{\mathrm{N}}^{\alpha}(q) \geqslant 0, \alpha \in \mathscr{I} \subset \mathbb{N}$ and Coulomb friction. The Newton-Euler formulation of such a system is given as:

$$
\left\{\begin{array}{ll}
\dot{q}=T(q) v, & \\
M \dot{v}=F(t, q, v)+H^{\top}(q) \lambda+G^{\top}(q) r, & \alpha \in \mathscr{E} \\
H^{\alpha}(q) v=0, & \widehat{u}^{\alpha}=u^{\alpha}+\mu^{\alpha}\left\|u_{\mathrm{T}}^{\alpha}\right\| \mathrm{N}^{\alpha} \\
u^{\alpha}=G^{\alpha}(q) v, & \text { if } g_{\mathrm{N}}^{\alpha}(q)>0, \\
r^{\alpha}=0, & \text { if } g_{\mathrm{N}}^{\alpha}(q)=0, \\
K^{\alpha, *} \ni \widehat{u}^{\alpha} \perp r^{\alpha} \in K^{\alpha} & \\
u_{\mathrm{N}}^{\alpha,+}=-e_{r}^{\alpha} u_{\mathrm{N}}^{\alpha,-}, & \text { if } g_{\mathrm{N}}^{\alpha}(q)=0 \text { and } u_{\mathrm{N}}^{\alpha,-} \leqslant 0,
\end{array}\right\} \alpha \in \mathscr{I}
$$

where $q$ is the vector of coordinates of the position and the orientation of the body, $v$ is the velocity, the operator $T(q) \in \mathbb{R}^{7 \times 6}$ links the time derivatives of the coordinates to the velocities, $M$ is the total inertia matrix, $F(t, q, v) \in \mathbb{R}^{6}$ collects all the forces and torques applied to the body. The operators $H \in \mathbb{R}^{m_{e} \times n}$ and $G \in \mathbb{R}^{3 m_{i} \times n}$ link the local velocity variables in the joints, and at contacts respectively, to the velocity vector of the bodies. 


\subsection{The Numerical Integration Method}

In this paper we use the event-capturing method based on the Moreau-Jean time-stepping scheme $[3,12,13]$, where the constraints are solved at the velocity level and thereafter named the NSCD method. It is well-known that velocity level treatment of constraints yields violations of constraints with the drift phenomenon. When we simulate mechanisms with small clearances, this is not tolerable since we have to keep the violation as small as possible with respect to the characteristic length of the clearances. To overcome this limitation of the standard Moreau-Jean time-stepping scheme, we use the combined projection scheme as proposed in [2].

\section{The C-60 Miniature Circuit Breaker Mechanism}

Miniature circuit breaker construction is simple, however very precise. In fact, a miniature circuit breaker has no replacement parts. It is not designed to be maintained. When a unit goes bad, it is simply replaced. A typical miniature circuit breaker mechanism is depicted in Fig. 3(a).

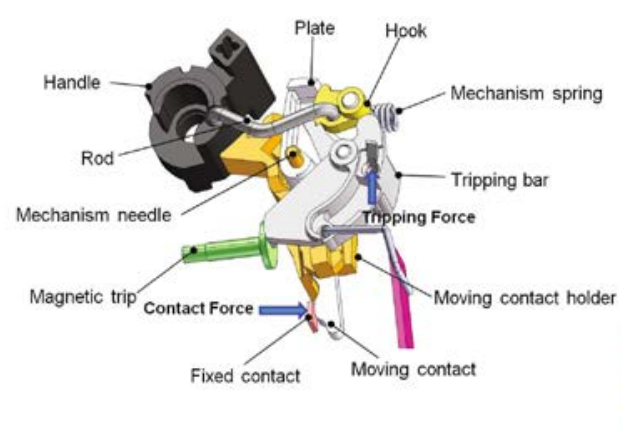

(a) $3 \mathrm{D}$ view.

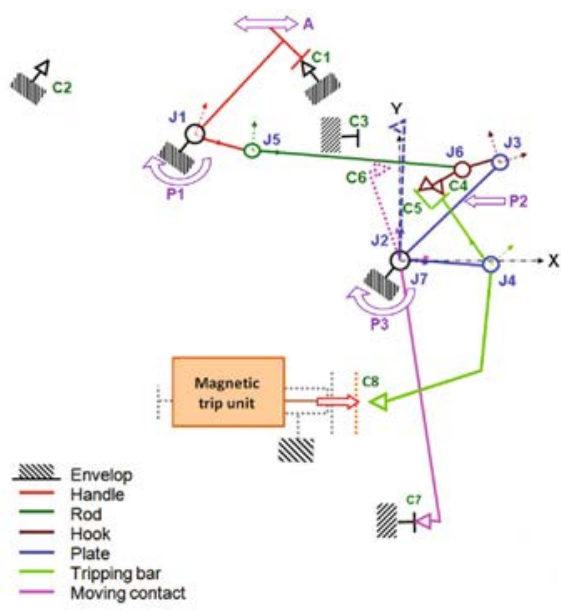

(b) Kinematic representation.

Fig. 3. C-60 circuit breaker mechanism - ON position.

Mechanism working principle: All the mechanism parts are enclosed inbetween the case and cover parts. These parts are connected to each other through a revolute joint or frictional contact. In the following section we will see the detailed description of these joints and contacts. In the first step, the primary function of a mechanism is usually formulated in terms of kinematical quantities (link geometry, kinematic constraints, etc). Also the various geometrical relations resulting from the kinematical analysis of the linkage mechanism 
are an essential ingredient for the dynamic analysis. The kinematical analysis of a miniature circuit breaker mechanism (hereafter called the $\mathrm{C}-60$ mechanism) is of great importance. The C-60 mechanism consists of seven links, seven revolute joints with clearance in both radial and axial direction and four frictional contacts (see Fig. 3(b)). It has 42 degrees of freedom. The operating mechanism in the ON position is explained as follows:

The close operation leads to ON position of the breaker. In close operation (see Fig. $3(\mathrm{~b})$ ), the operating handle $(A)$ is rotated clockwise which closes the contacts $C_{5}$ and $C_{4}$ through the revolute joints $J_{1}, J_{5}, J_{6}, J_{3}$ and $J_{4}$. The frictional contacts $C_{5}$ and $C_{4}$ have a specific wedge shape profile, which enables the locking between the hook and tripping bar. After the activation of the contacts $C_{5}$ and $C_{4}$ the motion has been transferred to the moving contact through the plate by revolute joints $J_{2}$ and $J_{7}$, which ensure closing of the contact between the moving and the fixed contact. During close operation the handle spring $\left(P_{1}\right)$ and the mechanism springs $\left(P_{2}\right.$ and $\left.P_{3}\right)$ get charged, which will be used for the trip operation of the breaker.

\subsection{Revolute Joint Between the Case, the Cover and the Handle: $J_{1}$}

The revolute joint between the case, the cover and the handle is $J_{1}$. The protrusion on the case and cover acts as a journal and the cavity on the handle acts as a bearing. In our modelling approach the ideal revolute joint is replaced by a journal and two circular rings at the extreme ends of the bearing (see Fig. 4) which acts as a spatial revolute joint with clearance. The axial and radial clearances in the revolute joint are modeled by introducing six degrees of freedom between the bearing and the journal. The relative motion between them is restricted by the internal surface of the bearing and the flanges of the journal. The radial clearance can be varied by changing the internal diameter of the bearing. The contact between the flange and the bearing top/bottom surface is a plane-plane contact. In reality, a plane-plane contact is impossible due to the presence of surface roughness and waviness. However the plane-plane contact can be completely described by three contact points. Few limitations of the plane-plane contacts are: more simulation time is required (numerically costly) as the contact detection is done on the entire area, and in return it gives only one contact point

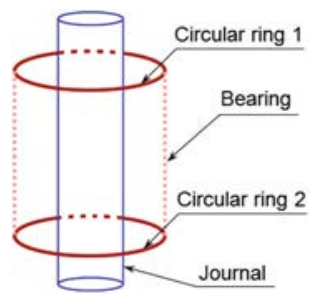

Fig. 4. Revolute joint with clearance $J_{1}$.

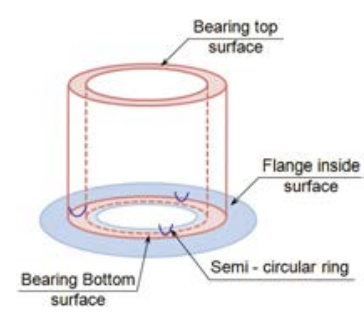

Fig. 5. Modeling of plane--plane contact. 
between the plane-plane contact which is practically not correct. The contact between the flange and the bearing face is modeled by considering the plane surface of the flange, while the plane surface of the bearing is replaced by three semi-circular equidistant rings (see Fig. 5).

\section{Experimental Validation: Contact Force Versus Displacement}

In this section we report comparisons between numerical results obtained with the NSCD method, and experimental data obtained on physical prototypes built by Schneider Electric. The radial clearance in the revolute joints is given as: $J_{1}=0.085 \mathrm{~mm}, J_{2}=0.05 \mathrm{~mm}, J_{3} / J_{4}=0.06 \mathrm{~mm}, J_{5} / J_{6}=0.045 \mathrm{~mm}$ and $J_{7}=$ $0.055 \mathrm{~mm}$. Referring to the arrow in Fig. 3, the comparisons are made by recording force and displacement histories at the moving contact. The test bench consists of the fixture to mount the $\mathrm{C}-60$ breaker and the moving table which comprises a pair of linear motion guide, see Fig. 6. The load cell is mounted on the moving table to measure the force and the bi-axial movement of the moving table is measured by two position sensors. The contact force of the moving contact $C_{7}$ (see Fig. 3(b)) is measured with the help of load cell, and is recorded by the computer programme. We have followed a similar methodology for the virtual testing (virtual test bench) of the C-60 product using the simulation.

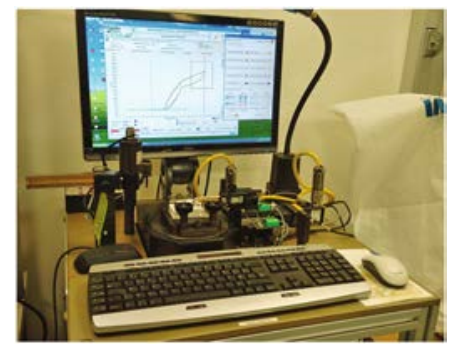

Fig. 6. Experimental test bench for contact/tripping force measurement.

1. In case of experimental test, the effect of polarization of the joints is approximately less by $50 \%$ when compared to the total displacement of the moving contact. At the static equilibrium (at the end of forward motion) of the sample-1, the recorded contact force is $15.28 \mathrm{~N}$ and the total displacement is $2.0 \mathrm{~mm}$, see Fig. 7(a). The results of the experimental test are compared with the numerical simulation. In case of the numerical test the effect of polarization is similar to that of the experimental test, see Fig. 7(b). However the trajectory of the contact force in both the experiment and numerical tests are not identical, this may be due to the fact that the geometrical variations on the contacting surfaces of the parts are not considered in the case of numerical simulation. In reality the geometrical variations always exist and these 


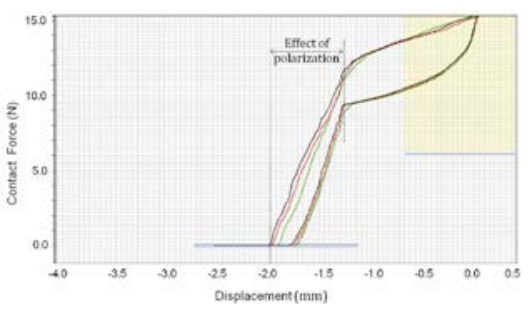

(a) Experimental result.

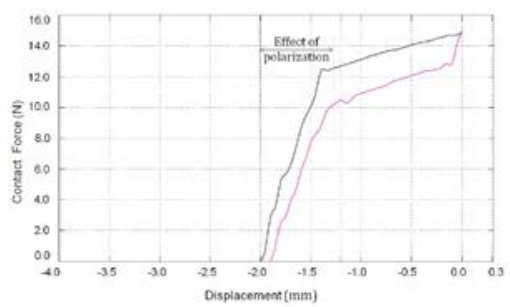

(b) Simulation result.

Fig. 7. Sample-1: Contact force versus displacement.

variations may change the polarization of the parts (contact points between the parts). In case of the numerical simulation, the contact force at the static equilibrium is $14.96 \mathrm{~N}$. The percentage relative error in the contact force between the experiment and numerical test is $2.08 \%$.

2. The trajectory of the contact force in case of forward motion is lagging behind the backward motion of the moving contact. This is due to the effects of friction (change in the direction of the frictional forces) in the joints. In case of the numerical simulation, the coefficient of friction between the plasticplastic materials is considered to be $\mu=0.3$, and between the steel-plastic materials $\mu=0.1$ In case of experimental test the real values of the coefficient of friction are not known. This may be one of the reasons behind the slightly different behaviour of the contact force trajectories between the experiment and virtual test.

\section{Conclusions}

This paper is devoted to the numerical simulation of the C-60 circuit breaker built by Schneider Electric, using the so-called Moreau-Jean NSCD eventcapturing numerical scheme. It relies on rigid body assumptions, with set-valued Coulomb's friction, and constant kinematic restitution coefficients. Emphasis is put on the modeling of three dimensional revolute joints with axial and radial clearance. Moreover detailed comparisons with experimental date obtained at the Schneider Electric laboratory, prove the very good prediction capabilities of the NSCD approach, for this type of mechanisms.

\section{References}

1. Abadie, M.: Dynamic simulation of rigid bodies: modelling of frictional contact. In: Brogliato, B. (ed.) Impacts in Mechanical Systems: Analysis and Modelling. Lecture Notes in Physics (LNP), vol. 551, pp. 61-144. Springer, New York (2000)

2. Acary, V.: Projected event-capturing time-stepping schemes for nonsmooth mechanical systems with unilateral contact and Coulomb's friction. Comput. Methods Appl. Mech. Eng. 256, 224-250 (2013) 
3. Acary, V., Brogliato, B.: Numerical Methods for Nonsmooth Dynamical Systems: Applications in Mechanics and Electronics. Lecture Notes in Applied and Computational Mechanics, vol. 35. Springer, Berlin (2008)

4. Acary, V., Cadoux, F., Lemarechal, C., Malick, J.: A formulation of the linear discrete Coulomb friction problem via convex optimization. ZAMM J. Appl. Math. Mech. (Zeitschrift für Angewandte Mathematik und Mechanik) 91(2), 155-175 (2011)

5. Akhadkar, N., Acary, V., Brogliato, B.: Analysis of collocated feedback controllers for four-bar planar mechanisms with joint clearances. Multibody Syst. Dyn. 38(2), 101-136 (2016)

6. Brogliato, B.: Nonsmooth Mechanics: Models, Dynamics and Control, 3rd edn. Springer International Publishing, Switzerland (2016)

7. De Saxcé, G.: Une généralisation de l'inégalité de Fenchel et ses applications aux lois constitutives. C. R. Acad. Sci. Ser. II 314, 125-129 (1992)

8. Flores, P.: A parametric study on the dynamic response of planar multibody systems with multiple clearance joints. Nonlinear Dyn. 61(4), 633-653 (2010)

9. Flores, P., Leine, R., Glocker, C.: Modeling and analysis of planar rigid multibody systems with translational clearance joints based on the non-smooth dynamics approach. Multibody Syst. Dyn. 23, 165-190 (2010)

10. Gummer, A., Sauer, B.: Influence of contact geometry on local friction energy and stiffness of revolute joints. J. Tribol. 134(2), 021402-1-021402-9 (2012)

11. Haroun, A., Megahed, S.: Simulation and experimentation of multibody mechanical systems with clearance revolute joints. In: Proceedings of World Academy of Science, Engineering and Technology, 63. World Academy of Science, Engineering and Technology (2012)

12. Jean, M.: The non-smooth contact dynamics method. Comput. Methods Appl. Mech. Eng. 177(3), 235-257 (1999)

13. Jean, M., Moreau, J.: Dynamics in the presence of unilateral contacts and dry friction: a numerical approach. In: del Piero, G., Maceri, F. (eds.) Unilateral Problems in Structural Analysis II. CISM Courses and Lectures, vol. 304, pp. 151-196. Springer, Vienna (1987)

14. Krinner, A., Thümmel, T.: Non-smooth behaviour of a linkage mechanism with revolute clearance joints. In: New Advances in Mechanisms, Transmissions and Applications, pp. 233-241. Springer (2014)

15. Orden, J.G.: Analysis of joint clearances in multibody systems. Multibody Syst. Dyn. 13(4), 401-420 (2005)

16. Thümmel, T., Funk, K.: Multibody modelling of linkage mechanisms including friction, clearance and impact. In: Proceedings of the 10th World Congress on the Theory of Machines and Mechanisms in Oulu, June 20 to 24, vol. 4, pp. 1387-1392. University Press, Finland (1999)

17. Yan, S., Xiang, W., Zhang, L.: A comprehensive model for 3D revolute joints with clearances in mechanical systems. Nonlinear Dyn. 80(1), 309-328 (2015) 